\title{
Possible therapeutic approach to apoptosis correction in nephrotic children
}

\author{
Burlaka Ie.A.* \\ Department of Pediatrics №4, O.O. Bogomolets National Medical University, Kyiv, Ukraine
}

\begin{abstract}
Objectives: the objective of this paper was to study the levels of apoptosis controlling factors and nuclear transcriptional factor NF- $\kappa \mathrm{B}$ in relation to the value of kidney function impairment; to evaluate possible protective effects of conventional therapy with addition of antioxidant agent tocopherol in pediatric patients with nephrotic syndrome.
\end{abstract}

Background: hypoxia-related disorders play an important role in irreversible kidney tissue damage via activation of certain secondary processes, i.e. apoptosis.

Methods: an examination of blood samples of 53 patients (aged 10 to 15 years) with nephrotic syndrome was done. Conventional clinical investigations, immunoblotting were used in this study.

Results: we show that nephrotic patients reveal a high level of pro-apoptotic marker Bax, low level of anti-apoptotic factor BcL-xL and anti-apoptotic unit of NF- $\kappa \mathrm{B}$ (p65). Their dependence on level of kidney function impairment was detected. Applied treatment composes conventional scheme and tocopherol shows a restored balance of apoptotsis controlling factor and NF- $\mathrm{B}$ activity.

Conclusion: hypoxia-induces disorders in nephrotic children resulted in apoptosis controlling system disturbances can be attenuated by the antioxidants application.

\section{Introduction}

Nephrotic-range proteinuria is the loss of 3 grams or more per day of protein into the urine or on a single spot urine collection, the presence of $2 \mathrm{~g}$ of protein per gram of urine creatinine. Nephrotic syndrome is the combination of nephrotic-range proteinuria with a low serum albumin level and edema.

Nephrotic syndrome has many causes, including primary kidney diseases such as minimal-change nephropathy, focal glomerulosclerosis, and membranous nephropathy. Nephrotic syndrome can also result from systemic diseases that affect other organs in addition to the kidneys, such as diabetes, amyloidosis, and lupus erythematosus.

Nephrotic syndrome may affect adults and children of both sexes and of any race. It may occur in typical form, or in association with nephritic syndrome. The latter connotes glomerular inflammation, with hematuria and impaired kidney function [1].

Main pathomorphological outcomes that apply to kidney damage in nephrotic syndrome are glomerulosclerosis, vascular sclerosis, tubule-interstitial fibrosis [2]. Inflammation plays an important role in the development and progression of chronic proteinuric kidney pathologies and is the primary and persistent violation, which underlies the pathogenesis of others. In addition to inflammation, fibrosis has a role in nephrotic syndrome. These disorders are accompanied by activation of the renin-angiotensin-aldosterone system, oxidative stress, endothelial dysfunction and others [1-3]. All mentioned above pathophysiological violations might be accompanied by apoptosis.

Apoptosis is programmed cell death that occurs when kidney disease has a place and plays an important role in their physiology. Harmful effects of apoptosis are in fact a source of a large number of kidney cells lost during and/or renal inflammation, scarring, loss of kidney function. The process of apoptosis is of fundamental importance to biological pathways ranging from embryogenesis to ageing and normal tissue homoeostasis to stress response. The most common form of NF- $\kappa \mathrm{B}$ is $\operatorname{RelA}(\mathrm{P} 65) / \mathrm{p} 50$ heterodimers, which are generally retained inert in the cytoplasm by the inhibitor protein, $\mathrm{I}-\mathrm{kappaB}(\mathrm{I} \kappa \mathrm{B})$. Following stimulation of the cell by a variety of agents, I $\kappa \mathrm{B}$ is degraded, allowing NF- $\kappa \mathrm{B}$ to translocate to the nucleus and bind to the promoter regions of its multiple target genes [4,5]. The molecular mechanisms underlying irreversible renal damage in children with nephrotic syndrome depending on apoptosis activation might be a potential therapeutic issue its treatment.

The main tasks in nephrotic syndrome treatment are renal insufficiency compensation, complications prevention and lowering the rate of the disease progression. Basic medicines for nephrotic syndrome treatment are immunoregulative drugs (corticosteroids, immunosuppressive agents), ACEi, Angiotensin receptors blockers. These pharmacologic manipulations that reduce urinary protein excretion in humans also limit progressive decline in renal function. Antihypertensive drugs have been used in humans to slow progression of renal disease in diabetic as well as non-diabetic glomerulopathies.

Correspondence to: Burlaka Ievgeniia. MD, $\mathrm{PhD}$, O.O. Bogomolets National Medical University, Department of Pediatrics №4, Kyiv, Ukraine, Tolstogo street 10, Tel: +38-097-432-49-44; E-mail: evgbur1982@gmail.com

Key words: treatment, tocopherol, nephrotic syndrome, HIlF-1 $\alpha, B a x, B c l-x L, N F$ $\kappa B$, immunoblotting

Received: February 28, 2017; Accepted: March 21, 2017; Published: March 24, 2017 
ACE inhibitors, for the same level of blood pressure control, are more renoprotective than other antihypertensives also used in human nephropathies, and this appears to be linked to their property of lowering urinary proteins to a greater extent than conventional drugs [6]. However, there are no specific non-toxic drugs regulating apoptosis and apoptosis-dependent disorders in this cohort of patients.

\section{Materials and methods}

\section{Patients}

An examination of renal biopsies of 53 patients (aged 10 to 15 years) with nephrotic syndrome hospitalized in Pediatric Nephrology unit of the Children Clinical Hospital №7 (Kyiv, Ukraine) was done. All patients were treated accordingly to the local protocols. Among all patients $24(45,28 \%)$ were with hormone-sensitive type of nephrotic syndrome, others - $29(54,72 \%)$ showed hormone-dependent type of nephrotic syndrome. Complex examination other than conventional methods (inspection, monitoring blood pressure, general and biochemical blood tests, determination of daily proteinuria, urinary sediment study and concentration ability of the kidneys, ultrasound of the abdomen etc.), immunohistochemical assessment of apoptosisdependent glomerular and tubule-interstitial damage were done.

The level of kidney function impairment (stage of Chronic Kidney Disease, CKD) was assessed by the value of glomerular filtration rate (GFR). GFR was calculated by Schwartz formula.

All patients were treated accordingly to local protocols which include corticosteroids (prednisolone), immunosuppressive drugs (CellCept, Leikeran), ACEi, diuretics (furosemide). An antioxidant (Vitamin E - tocopherol) beside mentioned above drugs in dose 100 $\mathrm{mg}$ /day was administered to patients from experimental group for 1 month.

\section{Immunoblotting for detection of Bax, Bcl-xL, NF-kB}

Proteins solubilized in Laemmli sample buffer were resolved in polyacrylamide gels by SDS-PAGE and transferred to a polyvinylidene difluoride membrane. Membranes were then blocked in 5\% non-fat milk in TBS-T (136 mM NaCl, $10 \mathrm{mM}$ Tris, $0.05 \%$ Tween 20 ) and immunoblotted using the Bax, Bcl-xL, NF-kB and HIF- $1 \alpha$ Ab (Cell Signaling Technology, Danvers, MA USA) and actin mouse mAb (BD, Lexington KY, USA) for 1 hour at room temperature. The actin mouse $\mathrm{mAb}$ was used as a loading control. After three washes with TBS-T, the membranes were incubated with secondary anti-rabbit or antimouse antibodies labeled with horseradish peroxidase for 1 hour at room temperature. Membranes were washed three times with TBS-T. The protein bands were visualized by chemiluminescent substrate ECL. Quantification of the protein content was done by densitometric analysis.

\section{Statistics}

Statistical analysis was done using the method of variation statistics (STATISTICA 6.0) and nonparametric statistical approaches (MannWhitney test). Results are presented as Mean \pm SEM. $\mathrm{P}<0.05$ was considered as statistically significant.

\section{Results}

\section{Apoptosis regulation in nephrotic children}

We have detected a decrease of anti-apoptotic factor BcL-xL levels. Detailed analysis shows that levels of BcL-xL decrease gradually depending on kidney function impairment. In nephrotic children with preserved kidney function BcL-xL levels were decreased to $75.1 \pm 2.2 \%$ in comparison to control values $(\mathrm{p}<0,01)$. In patients with CKD II-III decrease in BcL-xL documented as $60.1 \pm 1.8 \%$ of control value (та $\mathrm{p}<0,001$ ) (Figure 1).

In contrast, increased levels of pro-apoptotic factor Bax evaluated in all nephrotic children. Levels of Bax are dependent of kidney function. In patienst with CKD I Bax expression increased by $60.3 \pm$ $7.5 \%$ as compared to control $(\mathrm{p}<0,01)$ and by $90.1 \pm 9.8 \%(\mathrm{p}<0,001)$ in children with CKD II-III (Figure 2).

\section{NF-kB levels in nephrotic children}

We performed measurement of NF- $\mathrm{BB}$ (p65 subunit) that is responsible for protection against apoptosis. A pronounced decrease of its activity detected in all nephrotic children. In nephrotic patients with CKD I the level of NF- $\kappa B$ decreased by $15.2 \pm 5.9 \%$ as compared to control $(\mathrm{P}<0,01)$ and by $20.6 \pm 6.4 \%$ in nephrotic patients with $C K D$ II-III $(\mathrm{P}<0,001)$. Comparison between CKD I group and CKD II-III group doesn't show any statistical difference (Figure 3).

\section{Therapeutic correction of apoptotic process in children with nephrotic syndrome}

To evaluate the effectiveness of treatment in children with nephritic syndrome all patients were randomized and divided into three groups. The first group (main group) were children with nephrotic syndrome

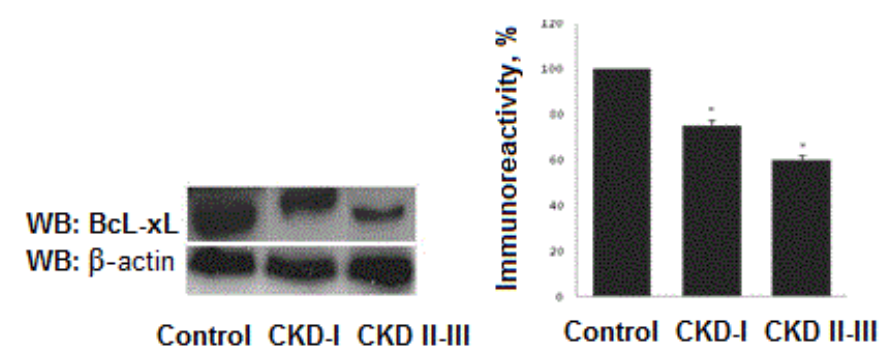

Figure 1. BcL-xL levels in children with nephrotic syndrome. ${ }^{*}$ - $<<0.05$.
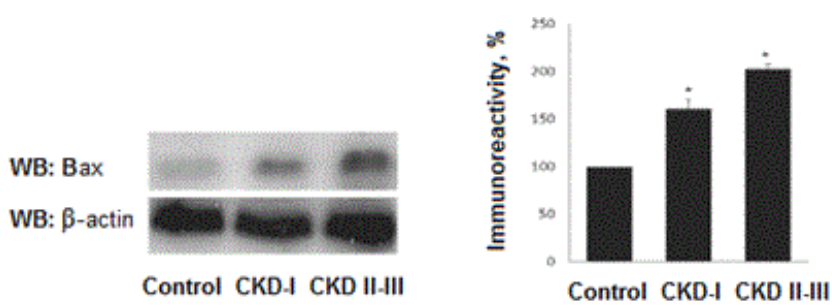

Figure 2. Bax levels in children with nephrotic syndrome.*- $<<0.05$.
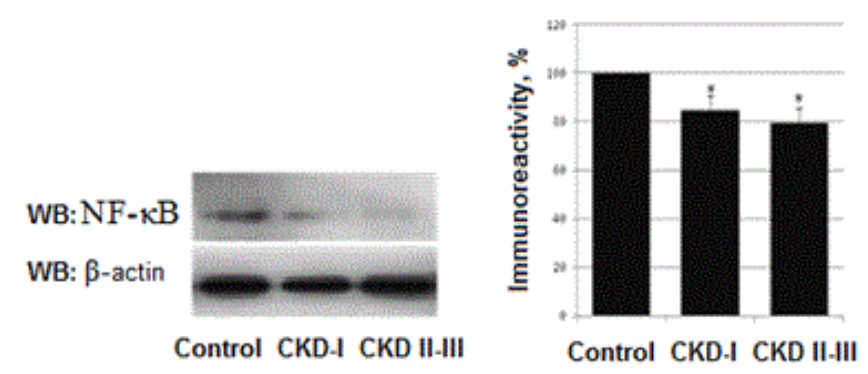

Figure 3. Levels of NF- $\kappa$ Bin nephrotic children. *- $\mathrm{P}<0.05$. 
who received conventional basic therapy. The second (experimental group) were children with nephrotic syndrome who received conventional basic therapy and tocopherol.The third group (control) included 45 healthy children matched by age and sex.

In our previous study we found that after the course of therapy all children investigated for serum level marker of chronic hypoxia HIFla show the decreased level as well as Bax leves [7].

Here we performend the measurement of serum levels of antiapoptotic factor $\mathrm{BcL}-\mathrm{xL}$. In children included to the main group $\mathrm{BcL}-\mathrm{xL}$ concentration was decreased to $81.7 .7 \pm 1.9 \%(\mathrm{p}<0.001)$ as compared to control. The control group value was taken as $100 \%$. After the course of treatment BcL-xL value was increased to $94.4 \pm 1.0 \%(\mathrm{p}<0.01)$ as compared to level before treatment. Children from experimental group exhibited BcL-xL before treatment at level $81.2 \pm 1.8 \%(\mathrm{p}<0.001)$ as compared to control group. After the course of treatment BcL-xL was up regulated to $87.25 \pm 1.6 \%$ ( $\mathrm{p}<0.01$ in comparison to the level before treatment) (Figure 4).

After the course of treatment all patients were analyzed for NF$\kappa \mathrm{B}$ levels. In patients of main group the level NF- $\kappa \mathrm{B}$ before treatment

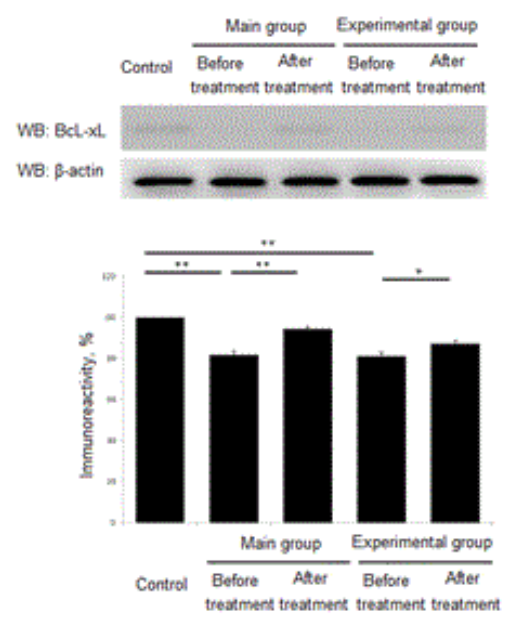

Figure 4. Treatment effect of anti-apoptotic factor BcL-xL. *- $\mathrm{P}<0.01 . \quad{ }^{*} *_{-} \mathrm{P}<0.001$.

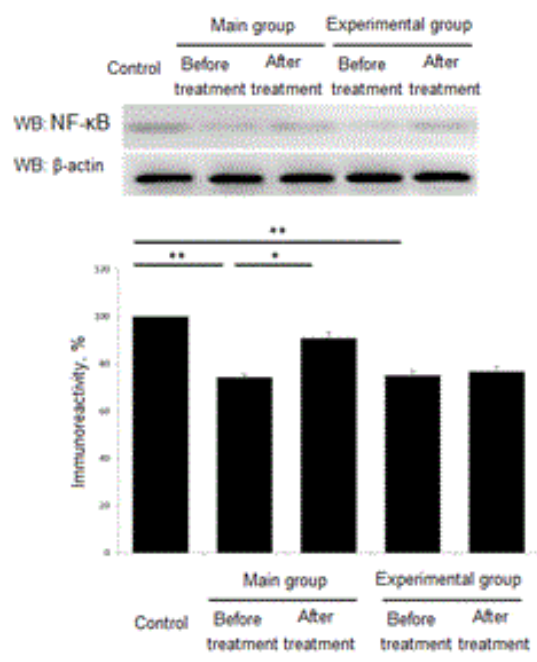

Figure 5. Treatment effect of anti-apoptotic factor NF- $\kappa B$. *- $\mathrm{P}<0.01$.**- $\mathrm{P}<0.001$. was down regulated to $74.2 \pm 1.06 \%$ in comparison to control group $(p<0,001)$. Control group value we set as $100 \%$. After the course of treatment the level of NF- $\kappa B$ increased up to $90.8 \pm 2.51 \%(\mathrm{p}<0,01)$. In children from experimental group level of NF- $\kappa B$ was down regulated to $75.1 \pm 1.98 \%(\mathrm{p}<0,001)$ as compared to control group. After the treatment we found a slight increase in NF- $\kappa B$ up to $76.7 \pm 2.17 \%$ $(\mathrm{p}>0,05)$. (Figure 5).

\section{Discussion}

Progression to irreversible renal parenchymal damage and endstage renal disease is the final common pathway of chronic proteinuric kidney disease and is relatively independent of the type of initial insult. In the past the amount of proteins found in the urine, taken as an indicator of the underlying abnormality in glomerular permeability, was considered by most nephrologists simply as a marker of the severity of renal lesions. Today the results of many studies indicate that proteins filtered through the glomerular capillary may have intrinsic renal toxicity, which together with other independent risk factors such as hypertension, can play a contributory role in the progression of renal damage $[1,5]$. Indeed, the secondary process of reabsorption of filtered proteins can contribute substantially to renal interstitial injury by activating intracellular events, including up-regulation of vasoactive and inflammatory genes, apoptosis activation. The corresponding molecules formed in excessive amounts by the renal tubules cause an interstitial inflammatory reaction that normally precedes renal scarring and correlates with declining renal function.

A reduction in proteinuria is associated with a slower decline in GFR. Enhanced albuminuria leads to the secondary pathologycal prosecces - inflammation, hypoxia, fibrosis [8]. Ischemia as a result of peritubular capillary loss or hypoperfusion is also considered a major factor for the progression of tubulointerstitial damage, which is closely associated with impairment of renal function. Renal tissue hypoxia induces profibrogenic responses and tubulointerstitial injury, which includes degeneration, dedifferentiation, cell death [9-11]. Microenvironmental changes, such as hypoxia, strongly impact inflammatory cell recruitment and function. Moreover, hypoxia has been shown to induce apoptosis, where HIF-1 plays a complex role. It has also been demonstrated that the expression of HIF-1 $\alpha$ significantly correlated with apoptosis and the pro-apoptotic factors, such as caspase-3, Fas, and Fas ligand. This finding has been shown on in vitro models [12].

Tocopherol is a fat-soluble antioxidant that is able to intercept free radicals in the plasma membrane, which helps to prevent the oxidative damage to lipids. It is known that patients with CKD have reduced levels of tocopherol in plasma, which is a prerequisite for this use in therapies [13]. Previous studies have shown that administration of tocopherol in patients with CKD helped to reduce the risk of cardiovascular complications, increased activity of endogenous antioxidant systems - GPX, catalase [14]. In our previous study [7] we have shown that treatment with an antioxidant lowered the hypoxiainduced cell damage as well as the elevation of HIF-1alpha in vitro [15]. Here we demonstrate that tocopherol administration has a potent effect on hypoxia-induced apoptosis development in children with nephrotic syndrome and subsequent restoration of the proapoptotic factor Bax, $\mathrm{BcL}-\mathrm{xL}$ and NF- $\kappa \mathrm{B}$ activation. We speculate that this therapeutical intervention may be a new non-toxic approach in apoptosis prevention in chronic albuminuric kidney pathologies.

\section{Acknowledgements}

This work has been done with support from nephrology unit of 


\section{Children Clinical Hospital №7 (Kyiv, Ukraine).}

\section{References}

1. Eddy AA, Symons JM (2003) Nephrotic syndrome in childhood. Lancet 362: 629-639. [Crossref]

2. Hemmelgarn BR, Manns BJ, Lloyd A, James MT, Klarenbach S, et al. (2010) Relation between kidney function, proteinuria, and adverse outcomes. JAMA 303: 423-429. [Crossref]

3. Certikova-Chabova V, Tesar V (2013) Recent insights into the pathogenesis of nephrotic syndrome. Minerva Med 104: 333-347. [Crossref]

4. Christman J.W, Blackwell T.S, Juurlink B.H.J (2010) Redox Regulation of Nuclear Factor Kappa B: Therapeutic Potential for Attenuating Inflammatory Responses. Brain Pathology 10: 153-162.

5. Nagel D, Vincendeau M, Eitelhuber AC, Krappmann D (2014) Mechanisms and consequences of constitutive NF-I ${ }^{\circ} \mathrm{B}$ activation in B-cell lymphoid malignancies. Oncogene 33: 5655-5665. [Crossref]

6. KDIGO 2012 Clinical Practice Guideline for the Evaluation and Management of Chronic Kidney Disease. Kidney Int Suppl 2013; 3:5.

7. Maidannyk V., Burlaka. Ie (2015) Management of Hypoxia-induced Disorders in Nephrotic Children. - J Cytol Histol 6: 36.

8. Gordillo R, Spitzer A (2009) The nephrotic syndrome. Pediatr Rev 30: 94-104. [Crossref]
9. Bento CF, Pereira P (2011) Regulation of hypoxia-inducible factor 1 and the loss of the cellular response to hypoxia in diabetes. Diabetologia 54: 1946-1956. [Crossref]

10. Eltzschig HK, Carmeliet P (2011) Hypoxia and inflammation. N Engl J Med 364: 656665. [Crossref]

11. Du R, Xia L, Ning X, Liu L, Sun W, et al. (2014) Hypoxia-induced Bmil promotes renal tubular epithelial cell-mesenchymal transition and renal fibrosis via PI3K/Akt signal. Mol Biol Cell 25: 2650-2659. [Crossref]

12. Volm M, Koomägi R (2000) Hypoxia-inducible factor (HIF-1) and its relationship to apoptosis and proliferation in lung cancer. Anticancer Res 20: 1527-1533. [Crossref]

13. Bin Q, Hu X, Cao Y, Gao F (2011) The role of vitamin E (tocopherol) supplementation in the prevention of stroke. A meta-analysis of 13 randomised controlled trials. Thromb Haemost 105: 579-585. [Crossref]

14. Young J.F, Rosenvold K, Stagsted J, Nielsen J.H, Andersen HJ (2005) Significance of vitamin E supplementation, dietary content of polyunsaturated fatty acids, and preslaughter stress on oxidative status in pig as reflected in cell integrity and antioxidative enzyme activities in porcine muscle. J Agric Food Chem 5: 745-749. [Crossref]

15. Bhatti F.U, Mehmood A, Latief N, Zahra S, Cho H, et al. (2017) Vitamin E protects rat mesenchymal stem cells against hydrogen peroxide-induced oxidative stress in vitro and improves their therapeutic potential in surgically-induced rat model of osteoarthritis. Osteoarthritis Cartilage 25: 321-331.

Copyright: (C2017 Burlaka Ie.A. This is an open-access article distributed under the terms of the Creative Commons Attribution License, which permits unrestricted use, distribution, and reproduction in any medium, provided the original author and source are credited. 